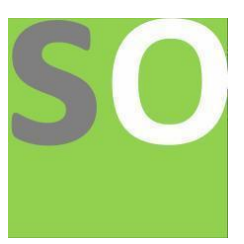

Article title: Is interleukin 6 measurement in exhaled breath of COVID-19 and post-COVID-19 lung fibrosis patients easy, sensitive and useful indicator of intensity of airway inflammation and fibrosis?

Authors: Amr Kamel Khalil Ahmed[1], Mahmoud El Kazzaz [2]

Affiliations: Director of tuberculosis program Ghubera, public health department, First health cluster, Ministry of health, Riyadh, Saudia Arabia[1], Department of chemistry and biochemistry, Faculty of Science, Damietta University, Egypt[2]

Orcid ids: 0000-0003-3477-236X[1], 0000-0003-3703-520X[2]

Contact e-mail: drmedahmed@gmail.com

License information: This work has been published open access under Creative Commons Attribution License http://creativecommons.org/licenses/by/4.0/, which permits unrestricted use, distribution, and reproduction in any medium, provided the original work is properly cited. Conditions, terms of use and publishing policy can be found at https://www.scienceopen.com/.

Preprint statement: This article is a preprint and has not been peer-reviewed, under consideration and submitted to ScienceOpen Preprints for open peer review.

Funder: NO

DOI: 10.14293/S2199-1006.1.SOR-.PPLARHX.v1

Preprint first posted online: 11 December 2021

Keywords: covid-19, interleukin-6, cytokine storm, exhaled breath, postcovid-19 lung fibrosis 


\title{
Is interleukin 6 measurement in exhaled breath of COVID-19 and post-COVID-19 lung fibrosis patients easy, sensitive and useful indicator of intensity of airway inflammation and fibrosis?
}

\author{
Amr Ahmed ${ }^{1}$ iD and Mahmoud Elkazzaz ${ }^{2}$
}

1. Director of tuberculosis program Ghubera, public health department, First health cluster, Ministry of health, Riyadh, Saudia Arabia. Orcid: 0000-00033477-236X

2. Department of Chemistry and Biochemistry, Faculty of Science, Damietta University, Egypt

3. Correspondence, Amr Ahmed. Email: drmedahmed@gmail.com

COVID-19 has emerged as a global pandemic. It mainly manifests as pneumonia, which may deteriorate into severe respiratory failure. The major hallmark of the disease is the systemic inflammatory immune response characterized by cytokine storm (CS). CS is marked by elevated levels of inflammatory cytokines, mainly interleukin-6 (IL-6), IL-8, IL-10, tumor necrosis factor- $\alpha$ (TNF- $\alpha$ ) and interferon- $\gamma$ (IFN$\gamma)$. Of these, IL- 6 is found to be significantly associated with higher mortality. IL- 6 is also a robust marker for predicting disease prognosis and deterioration of the clinical profile. (1)

IL-6 was detectable in the breath condensate of all healthy nonsmokers but was significantly higher in COPD patients. Exhaled breath condensate is totally noninvasive and highly acceptable to patients. The collection procedure has no effect on airway function or inflammation, and there is growing evidence that abnormalities in condensate composition may reflect biochemical changes in airway lining fluid. This method has been successfully used in previous studies to investigate several inflammatory markers in COPD and asthmatic patients. (2)

II-6 is produced in the lung by interstitial fibroblasts, alveolar macrophages, and large-vessel and bronchial epithelial cells. IL-6 levels are high in chronic inflammatory conditions of the lung, such as those due to allogeneic transplantation, bleomycininduced fibrosis and a variety of human interstitial lung diseases. High levels of IL- 6 have been found in the induced sputum of patients with COPD, particularly during exacerbation. Park et al. found increased IL-6 levels in the bronchioalveolar lavage fluid of patients with nonspecific interstitial pneumonia/fibrosis and in some patients with interstitial pneumonia. (3)

Dowlati et al. reported increased levels of IL-6 in the serum and BAL fluid of patients with lung cancer. (4) 
A recent study by Bhowmik et al. found increased IL-6 and IL-8 levels in the sputum of COPD patients with frequent exacerbations. (5)

Additionally, in another study, exhaled interleukin- 6 and leukotriene B4 levels may be useful noninvasive markers of airway inflammation in cigarette smokers. (6)

These studies and our novel method after clinical trials may open the field for future therapies for COVID-19 and post-COVID-19 lung fibrosis by inhaler transport medicines as a new challenge for overcoming sequelae of this pandemic. This suggested a new procedure for the measurement of exhaled IL-6. We studied which IL-6 is risky is IL-6 produced from airway or endocrine IL-6 or immune IL-6. We can classify IL- 6 as cytokine storm under pathological conditions to three mains of sources as immune IL- 6 produced from respiratory system like interstitial fibroblasts and bronchial epithelial cells and fibroblast, second endocrine IL- 6 produced from zona glomerulosa of adrenal glands stimulated by ACTH and immune IL-6 from macrophages and other immune cells.

From previous studies we need a procedure acceptable to patients and easy, noninvasive, sensitive so we plan for clinical trial registered at clinicaltrials.gov about measurement of interleukin- 6 at exhaled condensate of covid-19 patients and post-19 covid patients with lung fibrosis may be a useful tool, easy, sensitive for early intervention with anti-il6 medications and determine the degree of severity by inflammatory markers for intensity of airway inflammation.

Abbreviations:

IL-6: interleukin 6 
References:

1- Shekhawat J, Gauba K, Gupta S, Purohit P, Mitra P, Garg M, Misra S, Sharma P, Banerjee M. Interleukin-6 Perpetrator of the COVID-19 Cytokine Storm. Indian J Clin Biochem. 2021 Jun 21;36(4):1-11. doi: 10.1007/s12291-021-00989-8. Epub ahead of print. PMID: 34177139; PMCID: PMC8216093.

2- Bucchioni E, Kharitonov SA, Allegra L, Barnes PJ. High levels of interleukin- 6 in the exhaled breath condensate of patients with COPD. Respir Med. 2003 Dec;97(12):1299-302. doi: 10.1016/j.rmed.2003.07.008. PMID: 14682411.

3- C.S. Park, S.W. Chung, S.Y. Ki, et al. Increased levels of interleukin-6 are associated with lymphocitosis in bronchoalveolar lavage fluids of idiopathic nonspecific interstitial pneumonia Am J Resp Crit Care Med, 162 (2000), pp. 1162-1168.

4- A. Dowlati, N. Levitan, S.C. Remick Evaluation of interleukin-6 in bronchoalveolar lavage fluid and serum of patients with lung cancer J Lab Clin Med, 134 (4) (1999), pp. 405-409

5- A. Bhowmik, T.A. Seemungal, R.J. Sapsford, J.A. Wedzicha Relation of sputum inflammatory markers to symptoms and lung function changes in COPD exacerbations Thorax, 55 (7) (2000), pp. 629-630.

6- G.E. Carpagnano, S.A. Kharitonov, M.P. FoschinoBarbaro, O. Resta, E. Gramiccioni, P.J. Increased inflammatory markers in the exhaled breath condensate of cigarette smokers BarnesEuropean Respiratory Journal 2003 21: 589-593; DOI: 10.1183/09031936.03.00022203 